\title{
Failure to Increase Parathyroid Hormone Predicts Hypocalcemia After Neck Surgery for Endocrine Diseases: A Referral Center Experience With High Risk Patients
}

\author{
García-Sáenz Manuel¹, Mendoza-Zubieta Victoria', Mercado Moisés², \\ Ramírez-Rentería Claudia ${ }^{2 *}$ \\ ${ }^{1}$ Departamento de Endocrinología del Hospital de Especialidades "Dr. Bernardo Sepúlveda" Centro Médico Nacional Siglo XXI, Instituto \\ Mexicano del Seguro Social. Mexico City, Mexico. \\ ${ }^{2}$ Unidad de Investigación Médica en Endocrinología Experimental, Centro Médico Nacional Siglo XXI, Instituto Mexicano del Seguro Social. \\ Mexico City, Mexico.
}

Received: June 15,2019; Accepted: November 25,2019; Published: December 5,2019

*Corresponding author : Ramírez-Rentería Claudia, Clinical researched, Unidad de Investigación Médica en Endocrinología Experimental, Centro Médico Nacional Siglo XXI, Instituto Mexicano del Seguro Social. Avenida Cuauhtémoc N. 330, Col. Doctores, Del. Cuauhtemoc, 06720. Mexico City, Mexico. Tel: 5556276900 Ext21551. E-mail: clau.r2000@gmail.com

\begin{abstract}
Introduction

Transitory hypoparathyroidism occurs in 16.5-71\% of patients undergoing total thyroidectomy. Parathyroid hormone (PTH) rises quickly after serum calcium concentrations drop, before true hypocalcaemia ensues. We aimed to determine if this behavior is different in patients with or without hypocalcaemia after neck-surgery for endocrine diseases.
\end{abstract}

Methods

Total serum corrected calcium and intact PTH were determined before surgery and $6 \mathrm{~h}$ after, calcium determined at $12 \mathrm{~h}, 28 \mathrm{~h}$ and $24 \mathrm{~h}$ after surgery too, and compared between groups. Patients with other acute or chronic comorbidities that affect calcium metabolism were not included.

\section{Results}

83 patients, $86.7 \%$ women ( $47 \%$ premenopausal), with a median age of 52 years were evaluated. In total, $59 \%$ had biochemical hypocalcemia, while only $28.9 \%$ were symptomatic. A decrease of iPTH of greater than $9.5 \mathrm{pg} / \mathrm{mL}$ in the first 6 hours had a sensitivity of $100 \%$ and a specificity of 92.5\% to predict hypocalcemia. Discussion: most patients had a decrease in calcium during the first hours after surgery, but the patients without hypocalcemia had an appropriate increase of iPTH, while the patients that developed hypocalcemia had the opposite response. Conclusion: inappropriate reductions of iPTH in the first few hours after neck surgery for endocrine diseases, may be associated with hypocalcemia during the early postoperatory days. Clinical characteristics may be insufficient to detect the patients that will benefit from prophylactic calcium supplementation in some cases.

Keyword: Hypocalcemia; Thyroidectomy; Thyroid surgery; Parathyroid.

\section{Introduction}

Surgery accounts for $75 \%$ of the cases of hypoparathyroidism. The most frequently associated procedures include thyroid, parathyroid, laryngeal or other neck surgeries indicated for benign and malignant conditions. [1] It may be the result of parathyroid trauma, devascularization or inadvertent removal during the procedure. Other postulated explanations include haemodilution after aggressive fluid resuscitation, abnormal urinary calcium excretion, excessive calcitonin release or hungry bone syndrome. [2, 3]

Transitory hypoparathyroidism occurs in $16.5-71 \%$ of patients undergoing total thyroidectomy while permanent hypoparathyroidism is reported in only $1.5-1.8 \%$. [4, 5] Its true prevalence is underestimated due to the lack of clear definitions for hypocalcemia and normal reference values, the timing of blood sampling during the postoperative period, the length of follow-up and publication bias. [2]

Severe hypocalcemia can lead to serious complications; it may increase the length of hospitalization or even increase mortality. Several attempts have been made to detect or even predict hypocalcemia as early as possible, with contradictory results. $[4,6]$ Factors frequently associated with hypocalcemia are: low perioperative levels of serum calcium, 25-hydroxy-vitamin D (25(OH)D) and iPTH concentrations, failure to identify 2 or more parathyroid glands or reimplantation, the extent of the surgery, 
preoperative diagnosis of hyperthyroidism or malignancy and reoperation for bleeding. [7] Other factors may be age, gender or low alkaline phosphatase concentration.

Mexico is an iodine deficient area of the world where high incidences of goiter and other thyroid diseases are still present. Our population has a high prevalence of obesity, metabolic diseases and vitamin D deficiency and our hospital is a referral center for the most complicated cases. If all the patients in a single center are very similar and "high risk" according to the traditional risk factors described for hypocalcemia, additional tools are needed to detect those that will need calcium supplementation; given that the prophylactic use of calcium in all cases has not been proven to be cost effective either. Intact PTH determinations have been used extensively in parathyroid surgery as a biomarker for successful hyperparathyroidism surgery. It is very sensitive to small changes in serum calcium concentrations and it is able to shift serum calcium concentrations within minutes if necessary. The determination of iPTH is accessible for most surgical centers; it is cheap and results can be obtained quickly, making it a potentially useful biomarker. We hypothesized that the patients that underwent neck surgery for endocrine causes, may show different iPTH secretion patterns when they developed hypocalcemia, compared to the ones that did not, even when they were similar in most of the other clinical, biochemical or surgical characteristics.

\section{Materials and Methods}

We analyzed the files of adult patients who underwent neck surgery between July 2016 and June 2017 in our center. They were all treated by 4 experienced neck surgeons and the endocrinology department with more than 50 surgeries per year each. We collected the total serum calcium before surgery and $6 \mathrm{~h}, 12 \mathrm{~h}$, $18 \mathrm{~h}$ and $24 \mathrm{~h}$ after the procedure, and iPTH before and the first 6 hours after surgery corrected by albumin concentrations using the following formula: Corrected calcium=(4-Albumin $)^{*} 0.8+$ Measured total Calcium. Hypocalcemia was defined as a corrected total serum calcium $<8 \mathrm{mg} / \mathrm{dL}$ at any moment after surgery. We classified patients as having permanent hypoparathyroidism if they required oral calcium preparations to maintain normal serum calcium and iPTH was $<10 \mathrm{mg} /$ dL, 6 months after surgery. Calcium was supplemented with intravenous preparations of calcium gluconate between $0.5-2$ $\mathrm{mg} / \mathrm{kg} / \mathrm{hr}$ or oral $1 \mathrm{gr}$ calcium carbonate capsules, titrated every 12 hours according to laboratory results and considerations from the treating physician. In our center, all patients with a corrected calcium lower than $7.5 \mathrm{mg} / \mathrm{dL}$ or a decrease of $1.2 \mathrm{mg} /$ $\mathrm{dL}$ from the previous determination, are systematically started on oral calcium carbonate supplements and calcitriol $0.5-2 \mathrm{mg} /$ day as required. Starting doses are usually 1 gram of Calcium carbonate TID plus $0.25 \mathrm{mg}$ of calcitriol qd. We did not include patients with previous gastrointestinal diseases associated with malabsorption, advanced kidney or liver disease, recent events of proteinuria or malnutrition, sepsis, parenteral nutrition or that had been using other supplements or drugs that affect calcium metabolism in the 24 hours previous to the procedure. Patients that had been treated with anti-osteoporosis drugs in the last 6 months were not included either. We excluded patients that during surgery had severe blood loss that required aggressive fluid resuscitation, amines or transfusions, patients with fever or hypothermia, mechanic ventilation, acidosis or alkalosis. Patients with severe pain or other causes of altered respiratory patterns were excluded too.

During this period our hospital determined iPTH with a chemoluminiscent assay (DiaSorin Inc, Stillwater, Minnesota) with a sensitivity of $1 \mathrm{pg} / \mathrm{mL}$ and inter and intra-assay coefficient of variation (CV) of $5.3 \%$ and $3.5 \%$, respectively. Serum calcium and albumin were tested using automated methods based on colorimetric and enzymatic assays (COBAS, Roche). Statistical analysis was performed by a blinded investigator using SPSS version 23 and considering a $\mathrm{p}<0.05$ as significant. Descriptive data is expressed in medians and interquartile ranges (IQR).

\section{Results}

We reviewed a total of 123 files; forty did not meet the selection criteria. The remainder 83 patients were $86.7 \%$ women $(47 \%$ premenopausal), with a median age of 52 years (IQR 37-65). The median body mass index was $28.8 \%$, and only $17.3 \%$ had a BMI within normal ranges. The rest of the patients were classified as overweight (38.7\%) and $44 \%$ as obese (grade I in $30.7 \%$, grade II in $9.3 \%$ and grade III in $4 \%$ ). The main comorbidities were diabetes $(25.3 \%)$, hypertension (34.9\%), dyslipidemia (9.6\%), cardiopathy $(7.2 \%)$ or autoimmune diseases $(6 \%)$. All the patients had a $25(\mathrm{OH})$ vitamin D lower than $30 \mathrm{ng} / \mathrm{mL}$ and they were started on 1600 UI per day during their first pre-operative evaluation; however none of them reached normal levels of vitamin D at the time of surgery (after a median of 3 months).

A total of $59 \%$ of the patients had laboratory proven hypocalcemia, while only $28.9 \%$ had symptoms such as paresthesias, Chvostek or Trousseau's sign. The patients that required intravenous calcium supplementation required an average of $1 \mathrm{mg} / \mathrm{kg} / \mathrm{h}$ for 24 hours. The patients that required oral calcium were discharged from the hospital with a median dose of 4.8 grams (IQR 6-15) per day of elemental calcium (equivalent to 12 capsules of 1 gram of calcium carbonate), the mean calcitriol dose was $0.5 \mathrm{mcg} /$ day ( 2 capsules), only $12 \%$ required higher doses. Only $14 \%$ of the patients had a dose of 3 grams or less per day, which are the average doses reported to correct hypocalcemia in the literature. Only 1 patient was readmitted to the hospital due to hypocalcemia. The complete comparison between the groups with or without hypocalcemia can be observed in Table I.

When possible, the dose of oral calcium was reduced during the monthly follow up visits. A total of $16.3 \%$ had permanent hypoparathyroidism and at the last evaluation, $76.6 \%$ were taking doses of 3 grams/day or less, the rest continued to have high doses (between 4 and $40 \mathrm{~g}$ /day). The median hospital stay was 2 days (IQR 2-4); however, $25.3 \%$ of the patients had a stay of 4 days or longer because of hypocalcemia. 
There were no significant clinical differences between the patients with or without hypocalcemia at baseline, except for the tumor size if thyroid cancer was present $(\mathrm{p}<0.001)$. The decrease in calcium concentration ensued during the first 6 hours after surgery and it happened in both the patients who ultimately developed true hypocalcemia and those who didn't. The patients with hypocalcemia reached a calcium nadir during the first 12 hours after surgery. After the installation of treatment, the calcium concentrations returned to normal in the next 12-24 hours. We observed that in the patients without hypocalcemia, the calcium decrease was slower and milder than in the patients with hypocalcemia during the first 6 after surgery $(0.5 \mathrm{vs} 1.2 \mathrm{mg} /$ $\mathrm{dL}, \mathrm{p}=0.01$ ).

An important difference in the behavior of iPTH was detected between the two groups. The preoperative iPTH alone does not show a significant cutoff to predict hypocalcemia, but the measurement of iPTH after surgery is significantly lower in patients that developed hypocalcemia (12.5 vs $39.0, \mathrm{p}<0.001)$. Patients with a postoperatory iPTH higher than $25 \mathrm{pg} / \mathrm{mL}$ have a significantly lower probability of developing hypocalcemia (OR 0.041, CI 0.011-0.150, $\mathrm{p}<0.001$ ). The patients without hypocalcemia had a slight but significant increase of iPTH after surgery $(+1.91 \mathrm{pg} / \mathrm{mL}, \mathrm{p}=0.022)$, while the patients with hypocalcemia have a clear decrease in the hormone concentrations $(-25.04 \mathrm{pg} / \mathrm{mL}, \mathrm{p}<0.001$ ) (See Figure I) A decrease of iPTH of greater than $9.5 \mathrm{pg} / \mathrm{mL}$ had a sensitivity of $100 \%$ and a specificity of $92.5 \%$ to predict hypocalcemia with an area under the curve of 0.994 .

All the patients that underwent surgery to remove thyroid cancer tumor remnants had hypocalcemia $(n=5)$, while $67.2 \%$ of the patients that underwent thyroidectomy and $23.1 \%$ of the patients in the hemithyroidectomy group had the same complication. The patients that only had a neck dissection did not present hypocalcemia.

There was a previous diagnosis of cancer or suspicion of thyroid cancer in $52.4 \%$ of the patients which explains the extent of the surgeries. The patients who were diagnosed with cancer had nodules of approximately $2 \mathrm{~cm}$ (IQR 1-3.7), 46.2\% invaded the capsule and $31.6 \%$ invaded the surrounding tissues. They were classified as having high risk of recurrence in $36.8 \%$ of the cases using the American Thyroid Association risk score.

\section{Hypocalcemia}

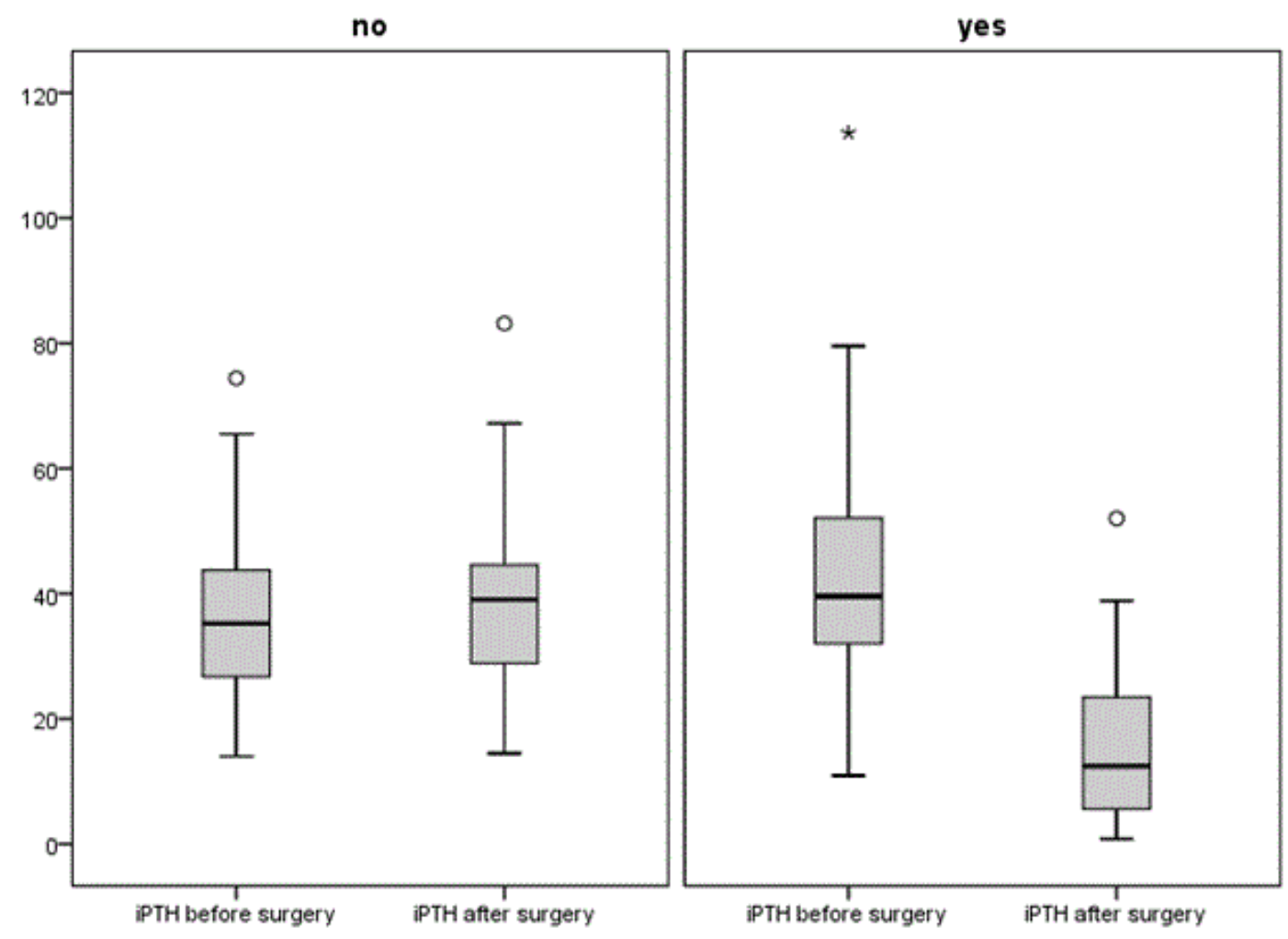

Figure I: Graphic of iPTH levels in both Groups. (iPTH = Intact Parathyroid Hormone). 


\begin{tabular}{|c|c|c|c|c|}
\hline & $\begin{array}{l}\text { Normal Range and } \\
\text { units }\end{array}$ & $\begin{array}{l}\text { Without hypocalcemia } \\
(\mathrm{n}=34)\end{array}$ & $\begin{array}{l}\text { With hypocalcemia } \\
(\mathrm{n}=49)\end{array}$ & $\mathrm{p}$ \\
\hline Age & -- & $45.5(34.8-62.0)$ & $54.0(38.5-66.5)$ & 0.166 \\
\hline Body Mass index & $18.5-24.99 \mathrm{~kg} / \mathrm{m} 2$ & $28.9(25.7-33.1)$ & $28.8(25.7-32.5)$ & 0.534 \\
\hline Female gender & -- & $79 \%$ & $91.8 \%$ & 0.115 \\
\hline Diabetes & -- & $29.4 \%$ & $22.4 \%$ & 0.609 \\
\hline Hypertension & -- & $26.5 \%$ & $40.8 \%$ & 0.243 \\
\hline Dyslipidemia & -- & $5.9 \%$ & $12.2 \%$ & 0.462 \\
\hline Obesity & -- & $41.2 \%$ & $38.8 \%$ & 0.826 \\
\hline Cardiovascular disease & -- & $5.9 \%$ & $8.2 \%$ & 1.000 \\
\hline Postmenopausal & -- & $25.0 \%$ & $43.5 \%$ & 0.109 \\
\hline Hyperthyroidism & -- & $8.8 \%$ & $2.0 \%$ & 0.300 \\
\hline Corrected total serum calcium (baseline) & $8.00-10.2 \mathrm{mg} / \mathrm{dL}$ & $9.0(8.9-9.5)$ & $9.0(8.7-9.3)$ & .198 \\
\hline Calcium $6 \mathrm{~h}$ after surgery & $8.00-10.2 \mathrm{mg} / \mathrm{dL}$ & $8.7(8.3-9.0)$ & $8.0(7.7-8.3)$ & $<0.001$ \\
\hline Calcium $12 \mathrm{~h}$ after surgery & $8.00-10.2 \mathrm{mg} / \mathrm{dL}$ & $8.7(8.4-9.0)$ & $8.0(7.8-8.6)$ & $<0.001$ \\
\hline Calcium $24 \mathrm{~h}$ after surgery & $8.00-10.2 \mathrm{mg} / \mathrm{dL}$ & $8.7(8.4-8.8)$ & $8.0(7.8-8.6)$ & $<0.001$ \\
\hline Calcium $48 \mathrm{~h}$ after surgery & $8.00-10.2 \mathrm{mg} / \mathrm{dL}$ & $8.6(8.5-8.9)$ & $8.7(8.0-9.3)$ & 0.896 \\
\hline Phosphorus & $2.7-4.5 \mathrm{mg} / \mathrm{dL}$ & $3.7(3.2-4.1)$ & $3.5(3.1-3.8)$ & 0.850 \\
\hline $25(\mathrm{OH})$ Vitamin D & $30-100 \mathrm{ng} / \mathrm{mL}$ & $17.7(9.0-26.1)$ & $16.5(12.1-20.9)$ & 0.975 \\
\hline iPTH before surgery & $10-65 \mathrm{pg} / \mathrm{mL}$ & $38.6(28.7-46.8)$ & $39.6(31.7-53.3)$ & 0.407 \\
\hline iPTH after surgery & $10-65 \mathrm{pg} / \mathrm{mL}$ & $39.0(27.0-44.3)$ & $12.5(5.6-23.5)$ & $<0.001$ \\
\hline Change in iPTH & $--\mathrm{pg} / \mathrm{mL}$ & 1.91 & -25.04 & $<0.001$ \\
\hline Alkaline phosphatase & $40-129 \mathrm{U} / \mathrm{L}$ & $82.5(56.8-86.8)$ & $78.0(64.0-98.0)$ & 0.360 \\
\hline Hospital stay & Days & $2(1-2)$ & $3(2.5-4)$ & $<0.001$ \\
\hline Surgery time & Hours & $2.9(2.1-3.7)$ & $3.6(2.3-4.7)$ & 0.040 \\
\hline Fluid balance during surgery & Milliliters & $65(-120-215)$ & $70(-86-350)$ & 0.589 \\
\hline Maximum tumor diameter (if thyroid cancer present) & Centimeters & $1.1(0.4-2.0)$ & $2.0(1.4-4.0)$ & 0.019 \\
\hline
\end{tabular}

\section{Discussion}

Even when hypoparathyroidism is considered to be a common and frequently transitory complication of neck surgery that is related to the surgeon's experience, there are other factors that may influence this result. [8, 9] Patients with advanced age, postmenopausal women, extensive surgeries, especially with neck dissections, history of long- standing hyperthyroidism, diabetes, obesity, history or suspicion of malignancy as well as the type of surgery, the total number of parathyroid glands identified during operation and calcium variations are some of the factors associated with this outcome. However, there are still no guidelines that can help clinicians to safely treat all patients using only these pointers to predict hypocalcemia. These contradictions may arise from the heterogeneity of surgeries evaluated, the laboratory pitfalls, the time points of evaluation and definitions for hypocalcemia, etc. Even when hypocalcemia may be mild and transient in most cases, it is potentially deadly and costly if treated incorrectly (either under or overtreated), and centers with a high volume of patients need to have additional markers to help them treat their patients correctly. This may require that general algorithms are adapted to each centers resources and expertise, but we consider that high risk patients, such as ours, can benefit from the determination of iPTH before and early after surgery. We should notice that many of these patients had normal PTH levels in all the evaluations, despite Vitamin D deficiency, and some of them even had mildly elevated iPTH. The iPTH had small changes that may go unnoticed precisely because they remain "normal" and only few (14 patients, only 23\% of patients with hypocalcemia) of them had iPTH under $15 \mathrm{pg} / \mathrm{mL}$ (true hypoparathyroidism). But even when they started with normal PTH and the reduction was small, this is an inappropriate response to the declining calcium concentrations, which should be detected by the parathyroid glands swiftly. The patients that did not develop true hypocalcemia also showed a decline in serum calcium within the first hours after surgery, but their parathyroid glands seem to detect these changes and maintain or slightly increase iPTH production in compensation. We can only speculate as to the causes of these differential responses, given that the patients were very similar in many other aspects. It could be that these patients had a stunned iPTH response due to 
surgical manipulation, but it may also reflect different set points for iPTH secretion in each patient or the presence of additional causes for altered vitamin D metabolism.

Our series has a high frequency of hypocalcemia even with experienced surgeons, which we attribute to the severity of the diseases treated in a referral center, along with the previously mentioned factors.

Calcium metabolism is finely regulated by very different and ever changing factors, some of which may be modifiable and others that may not. Both vitamin D and iPTH are usually low in patients with obesity or chronic diseases and it is possible that patients with these characteristics may have altered calcium metabolism dynamics. [1, 10] Our patients were mostly 50 years old, overweight or obese women, with a high probability of having thyroid cancer and other comorbidities. This may explain why all of them have severe vitamin D deficiencies that are not easily corrected with the recommended dose of vitamin D supplements and why they were candidates to aggressive neck surgeries. We should also remember that neck fat may be difficult to manipulate during surgery and anesthetic procedures may take longer, which also increases the probability of hypocalcemia. We didn't find any significant differences in age, sex, or comorbidities between the patients with or without hypocalcemia, unlike other authors that found that an age $>57$ years, diabetes or the female were more predictive of hypocalcemia. $[11,12]$ This may be because most of our patients have those characteristics, which makes it difficult to find differences among them.

Interestingly, we found that a single iPTH determination, decreased calcium concentrations or vitamin D, [13-17] were not enough to predict hypocalcemia; but rather, it is the change in iPTH concentrations that may predict the calcium's behavior. [18, 19] We observed that most patients had a decrease in calcium during the first hours after surgery, the expected response of a parathyroid gland with a normal function would be to increase the secretion of iPTH to prevent hypocalcemia, and it may not be a false positive as previously considered. [20] This "appropriate iPTH response" is present in the patients that consequently didn't present hypocalcemia, while the patients that not only didn't increase iPTH but even decreased their concentrations, eventually presented hypocalcemia, reflecting a greater damage to the parathyroid gland and a delayed or absent response to hypocalcemia. In our study a decrease of $9.5 \mathrm{pg} / \mathrm{mL}$ or more in PTH concentrations had a good sensitivity and specificity to predict hypocalcemia, this means that patients may still have normal or detectable serum iPTH, but it is not enough to counter regulate the rapid fall in calcium. We hypothesize that an insufficient increase of iPTH levels in these cases may be caused by parathyroid glands that are synthetizing the hormone but at a lower speed than needed or that the hormone is not able to reach the general circulation fast enough after its vascular tissue has been damaged by surgery.

Finally, the need for large doses of calcium supplements in our patients is remarkable. Scientific literature suggests that 3 grams of elemental calcium per day is the median dose required to control surgical hypoparathyroidism. [5, 6] Our patients required around 4.8 grams per day, which is roughly $60 \%$ more than the average dose reported for other series. We consider that the vitamin D deficiency may be partially responsible for this variation. The literature suggests that the patients with vitamin D deficiency should be supplemented with 5000 to 10,000 UI/ day to achieve normal concentrations before surgery; however in our country, the highest dose available commercially until a few months ago was $1600 \mathrm{UI}$ and these supplements are not freely available for the patients in our institution. Considering that serum $25(\mathrm{OH})$ vitamin D determinations are still expensive in some centers and not available in general hospitals or primary care facilities, supplementing the patients with vitamin D prior to surgery may pose an additional problem. High doses may not be a safe choice in most cases unless toxicity can be closely monitored, but patients with excess weight or with comorbidities associated with low vitamin D may benefit from doses that do not go over $10000 \mathrm{UI} /$ day to avoid toxicity as recommended by international guidelines. [21]

\section{Conclusion}

Post surgery hypocalcemia may be multifactorial and more difficult to prevent and treat in high risk groups. An inappropriate decrease of iPTH of $>9.5 \mathrm{pg} / \mathrm{dL}$ after surgery may be associated with hypocalcemia in the first postoperatory hours. We suggest that vitamin D supplementation before surgery and treatment with prophylactic calcium may be useful in such cases (See Figure II).

\section{Acknowledgements}

This job did not receive any financial support.

\section{Declarations}

None of authors have any conflict interest. 


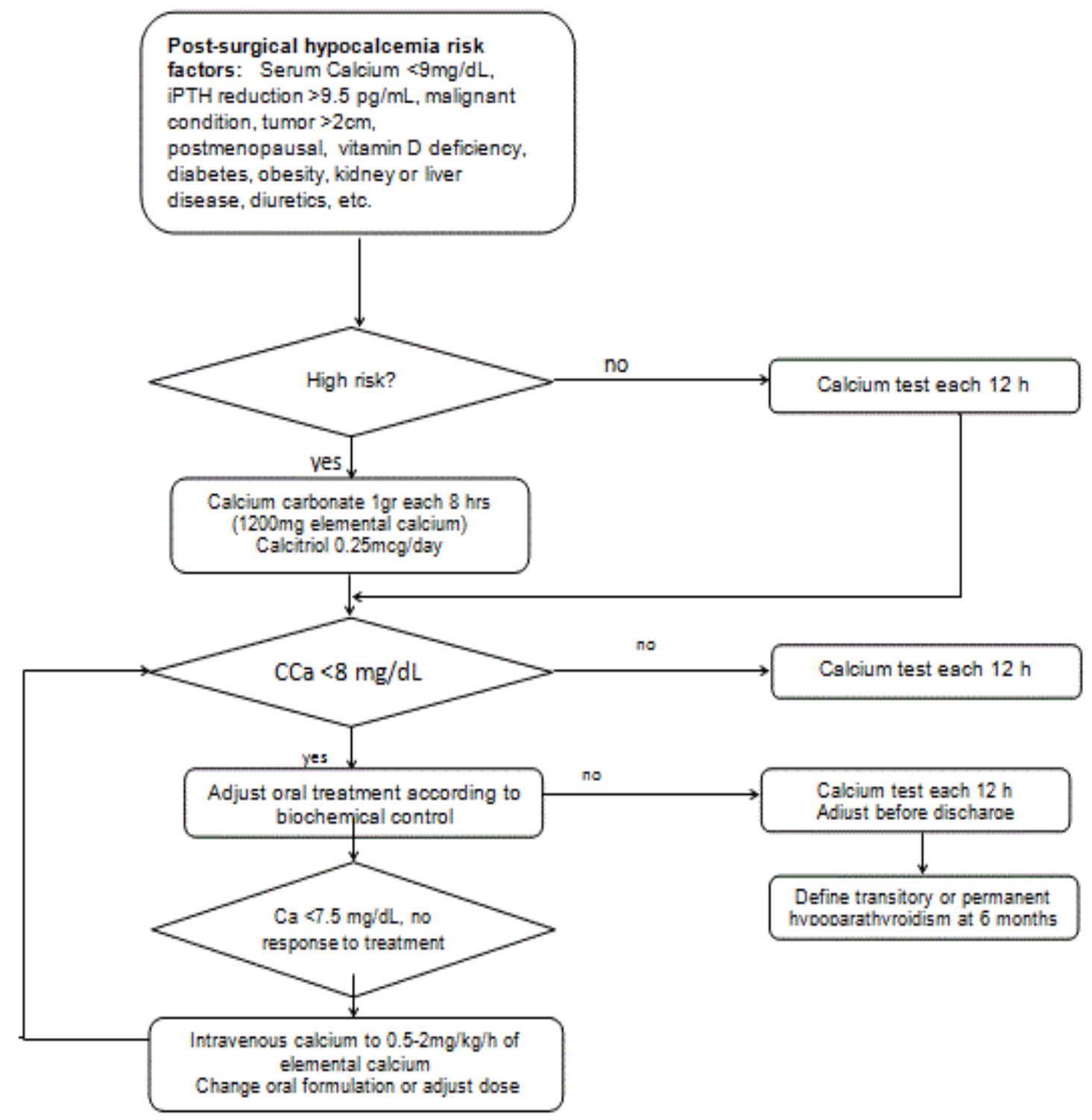

Figure II: Algorithm propose to post-surgical thyroid followed. (iPTH = Intact Parathyroid Hormone,CCa = Corrected Serum Calcium).

\section{References}

1. Dolores M. Shoback, JPB, Aline G. Costa, David Dempster, Henning Dralle, Aliya A. Khan, et al. Presentation of Hypoparathyroidism: Etiologies and Clinical Features. The Journal of clinical endocrinology and metabolism. 2016;101(6):2300-2312.doi:10.1210/jc.2015-3909

2. Palermo A, Mangiameli G, Tabacco G, Longo F, Pedone C, Briganti SI, et al. PTH(1-34) for the Primary Prevention of Postthyroidectomy Hypocalcemia: The THYPOS Trial. J Clin Endocrinol Metab. 2016;101(11):4039-4045.

3. MS Islam, DP, T Sultana, MQ Rahman, Z Rehena, ANN Ahmed. Evaluation of Serum Calcium Level Measurement in Total Thyroidectomy Patients - A Prospective Study in Tertiary Hospitals. Bangladesh J Med Biochem. 2011;4(1):4-9. doi:10.3329/bjmb.v4i1.13775
4. Rosa KM, Matos LL, Cernea CR, Brandao LG, Araujo Filho VJ. Postoperative calcium levels as a diagnostic measure for hypoparathyroidism after total thyroidectomy. Arch Endocrinol Metab. 2015;59(5):428-433.doi:10.1590/2359-3997000000074

5. Alhefdhi A, Mazeh H, Chen H. Role of postoperative vitamin $\mathrm{D}$ and/or calcium routine supplementation in preventing hypocalcemia after thyroidectomy: a systematic review and meta- analysis.Oncologist.2013;18(5):533-42.doi: 10.1634/ theoncologist.2012-0283

6. Singer MC, Bhakta D, Seybt MW, Terris DJ. Calcium management after thyroidectomy: a simple and cost-effective method. Otolaryngol Head Neck Surg. 2012;146(3):362-365. doi: 10.1177/0194599811433557

7. Michael Mannstadt, John P. Bilezikian, Rajesh V. Thakker, Fadil M. 
Hannan, Bart L. Clarke, Lars Rejnmark, et al. Hypoparathyroidism. Nature reviews disease primers.2017; 3 (17055):1-20.

8. Brandi ML, Bilezikian JP, Shoback D, Bouillon R, Clarke BL, Thakker RV, et al. Management of Hypoparathyroidism: Summary Statement and Guidelines. J Clin Endocrinol Metab. 2016;101(6):2273-2283. doi: 10.1210/jc.2015-3907

9. Harris AS, Prades E, Tkachuk O, Zeitoun H. Better consenting for thyroidectomy: who has an increased risk of postoperative hypocalcaemia? Eur Arch Otorhinolaryngol. 2016;273(12):44374443. doi: 10.1007/s00405-016-4084-4

10. Shoback DM, Bilezikian JP, Costa AG, Dempster D, Dralle H, Khan AA, et al. Presentation of Hypoparathyroidism: Etiologies and Clinical Features. J Clin Endocrinol Metab. 2016 Jun;101(6):2300-2312. doi: 10.1210/jc.2015-3909

11. Erbil Y, Barbaros U, Temel B, Turkoglu U, Issever H, Bozbora A, et al.The impact of age, vitamin $\mathrm{D}(3)$ level, and incidental parathyroidectomy on postoperative hypocalcemia after total or near total thyroidectomy. Am J Surg. 2009;197(4):439-446. doi: 10.1016/j.amjsurg.2008.01.032

12. Al-DhahriSF, Mubasher M,Al-Muhawas F, Alessa M, Terkawi RS, Terkawi AS. Early Prediction of Oral Calcium and Vitamin D Requirements in Post-thyroidectomy Hypocalcaemia. Otolaryngol Head Neck Surg. 2014;151(3):407-414. doi: 10.1177/0194599814536848

13. C. Gopalakrishnan Nair, Misha J. C. Babu, Riju Menon, Pradeep Jacob. Hypocalcaemia following total thyroidectomy: analysis of 806 patients. Indian J Endocrinol Metab. 2013; 17(2): 298-303. doi: 10.4103/22308210.109718

14. Barquero MH, Delgado RMJ, Juantá JC. Hipocalcemia e hipoparatiroidismo post-tiroidectomía. Acta Médica Costaricense. 2015; 57 (4): 184-189.
15. Noureldine SI, Genther DJ, Lopez M, Agrawal N, Tufano RP. Early Predictors of Hypocalcemia After Total Thyroidectomy. An Analysis of 304 Patients Using a Short-Stay Monitoring Protocol. JAMA Otolaryngol Head Neck Surg. 2014;140(11):1006-1013. doi: 10.1001/ jamaoto.2014.2435

16. AlQahtani A, Parsyan A, Payne R, Tabah R. Parathyroid hormone levels 1 hour after thyroidectomy: an early predictor of postoperative hypocalcemia. Can J Surg. 2014;57(4):237-240.

17. Lombardi CP, Raffaelli M, Princi P, Santini S, Boscherini M, De Crea C, et al. Early prediction of Postthyroidectomy hypocalcemia by one single iPTH measurement. Surgery. 2004;136(6):1236-1241.

18. Lecerf P, Orry D, Perrodeau E, Lhommet C, Charretier C, Mor C, et al. Parathyroid hormone decline 4 hours after total thyroidectomy accurately predicts hypocalcemia. Surgery. 2012;152(5):863-8. doi: 10.1016/j.surg.2012.03.011

19. De Pasquale L, Sartori PV, Vicentini L, Beretta E, Boniardi M, Leopaldi E, et al. Necessity of therapy for postthyroide- ctomy hypocalcaemia: a multi-centre experience. Langenbecks Arch Surg.2015;400(3):31924. doi: 10.1007/s00423-015-1292-0

20. Mathur A, Nagarajan N, Kahan S, Schneider EB, Zeiger MA. Association of Parathyroid Hormone Level With Postthyroidectomy Hypocalcemia A Systematic Review. JAMA Surg. 2018;153(1):69-76. doi: 10.1001/ jama surg.2017.3398

21. Holick MF, Binkley NC, Bischoff-Ferrari HA, Gordon CM, Hanley DA, Heaney RP, et al. Evaluation, Treatment, and Prevention of Vitamin D Deficiency: an Endocrine Society Clinical Practice Guideline. J Clin Endocrinol Metab. 2011; 96(7):1911-30. doi: 10.1210/jc.2011-0385 